\title{
An adaptive method for health trend prediction of rotating bearings
}

\author{
Sheng Hong ${ }^{\mathrm{a}, *}$, Zheng Zhou ${ }^{\mathrm{b}}$, Enrico Zio ${ }^{\mathrm{c}, \mathrm{d}}$, Wenbin Wang ${ }^{\mathrm{e}}$ \\ a Science E' Technology Laboratory on Reliability \& Environmental Engineering, School of Reliability and System Engineering, Beihang University, Beijing, China \\ b Systems Engineering Research Institute, China State Shipbuilding Corporation (CSSC), China \\ c Department of Energy Polytechnic of Milan, Via Ponzio 34/3, 20133 Milan, Italy \\ d Ecole Central Paris et Supelec, Paris, France \\ e Dongling School of Economics and Management, University of Science and Technology Beijing, Beijing, China
}

Available online 27 August 2014

\section{Introduction}

Prognostics and health management (PHM) is expected to provide early detection of incipient faults and predict the progression of degradation in industrial components and systems [1-3]. Condition monitoring (CM) data, such as vibration, temperature, and pressure are collected and techniques of signal processing, feature extraction, health assessment, and RUL prediction are developed to fulfill the goals of a PHM system [4].

Rotating bearings are very common mechanical components and play an important role in a number of industrial applications. In many instances, operation of these components is in harsh working and environmental conditions, which can lead to unex-pected failures [5]. In order to avoid fatal breakdowns and the consequent decrease of machinery service performance, effective component and system health management, and accurate remain-ing useful life (RUL) prediction are interesting solutions to imple-ment while the roller bearing is operating.
Bearing as a common rotary machinery component, has attracted attention in both industry and academia [6-8]. The research efforts in the area of PHM for bearings have resulted in the development of various algorithms and models tailored to specific applications. With the spread of artificial intelligence and machine learning technologies, data-driven methods for estimating the RUL based on CM data have gained attention for rotating bearing health management. Heng et al. made a review of prognostics techniques and current challenges for rotating machinery prognosis [9]. Si et al. systematically reviewed the data-driven models and approaches reported in the literature in recent decades [10]. Benkedjouh et al. proposed the use of the isometric feature mapping reduction technique (ISOMAP) and support vector regression (SVR) for degradation assessment and RUL prediction [11]. Zhao Wei et al. utilized a dynamic particle filter-support vector regression method for reliability prediction [12].

Each of these prognostics models proposed in the literature has good result, however, a single prediction model may not be able to handle all situations in real practice $[7,13]$. Recently, researchers focus on the adaptive prognostics strategy in order to get a better prediction results. Liu points out the importance to balance the prediction efficiency and accuracy adaptively and propose an

\footnotetext{
* Corresponding author. Address: No. 631 Weimin Building, \#37 Xue yuan Rd., Haidian district, Beihang University, Beijing 100191, China.

E-mail address: fengqiao1981@gmail.com (S. Hong).
} 
on-line adaptive data-driven prognostics strategy of SVR method [14]. Liao and Kottig applied a hybrid prognostics method to a battery degradation case to show the potential benefit of the hybrid approach [15]. Liao and Tian provide a framework for predicting the RUL under time-varying operating conditions [16]. Sun et al. develop a state-space-based degradation model to reduce failure prognostics uncertainty [17]. Bearing degradation has great uncertainty and the dynamic degradation states have significant influence on the PHM models effectiveness $[18,19]$. Although there are some adaptive methods, which can adjust their modeling by changing the parameters to follow different degradation dynamics, the results are not always satisfactory under some other circumstance $[20,21]$. Furthermore, the adequacy of the model for the different dynamic stages of degradation should also consider the time requisites of the application and the effects of algorithmic complexity $[22,23]$. In fact, in some applications CPU computing resources may be limited frequently in industrial machinery operation $[24,25]$. The control computers are running their own working programs and they leave not too much computing resources for PHM algorithms. Therefore, reducing the computational complexity while ensuring accuracy, can be particularly important in practice, especially for industrial systems with limited computa-tional resources. Alternatively, a condition-based method could be developed capable of selecting the adequate prognostic models depending on the current dynamic condition state of the bearing.

Rotating bearing degradation is a physical process that typically evolves dynamically in stages characterized by different speeds of evolution of the characteristic health indicators. Therefore, it is op-portune to apply different predictive models in the different stages, with the aim of balancing accuracy and calculation complexity. This leads to an adaptive scheme of PHM, whereby the stages in which the degradation proceeds gracefully calls for methods with less accuracy and, therefore, less computationally demand-ing, whereas the stages in which the process evolves faster call for more accurate predictions but at the expense of more demand-ing efforts in computation. In this way, the adaptive approach can select the better algorithm according to the varying degradation stages, while avoiding the limitations of a single algorithm.

To cope with the dynamic degradation behavior of rotating bearings and choose the proper prognostics methods for life pre-diction, an adaptive method for health assessment and prognosis is proposed in this paper, based on the analysis of vibration signals. The original acceleration vibration signal is decomposed by empirical mode decomposition (EMD) and the useful intrinsic mode functions (IMF) are obtained. Then, the EMD energy entropy, which can reflect the actual health condition, is converted into a con-fidence value $(\mathrm{CV})$ to assess the bearing health state, by using a SOM method. In order to dynamically select the proper prognostics models, the bearing health state is categorized into four different health stages to each of which corresponds a specific method for predicting the health trend. A case study of a bearing run-to-failure test is analyzed.

The paper is organized as follows. Section 2 describes the bearing health assessment method based on EMD energy entropy, and SOM. The bearing health state is represented by the computed CV. Section 3 presents the framework of the proposed adaptive prediction method. In Section 4, the experimental verification and results are presented with reference to the bearing run-to-failure test. The conclusion of this paper is given in Section 5.

\section{Health assessment}

\subsection{EMD energy entropy}

EMD is a powerful signal processing technique, extensively studied and applied in prognostics of rotating bearings [26].
Traditional signal processing techniques, including timedomain and frequency-domain analysis, cannot provide complete information of the vibration signals of the bearing, which possess non-stationary and non-linear characteristics. As a selfadaptive method for time-frequency analysis, EMD is here adopted to decompose the signal into a number of IMFs and the residue of the decom-position [27]. The original signal $x(t)$ is decomposed in terms of $n$-empirical modes as follows,

$x(t)=\sum_{i=1}^{n} c_{i}(t)+r_{n}(t)$

where $r_{n}(t)$ is the residual function and $c_{i}(t)$ are the IMFs of different frequency bands ranging from high to low.

While the roller bearing is operating under different working conditions, the energy of the signal changes with the frequency distribution. The EMD energy entropy is used to illustrate the change of energy. For a vibration signal $x(t)$, the $n$ IMFs and the residue $r_{n}(t)$ are obtained by using (1): the energies of the $n$ IMFs $E_{1}, E_{2}, \cdots, E_{n}$, are calculated as follows,

$E_{i}=\int_{-\infty}^{+\infty}\left|c_{i}(t)\right|^{2} d t \quad(i=1,2, \ldots, M)$

and the corresponding EMD energy entropies are designated as,

$H_{E N}=-\sum_{i=1}^{M} p_{i} \log p_{i}$

where $p_{i}=E_{i} / E$ ( $\left.E=\sum_{i=1}^{n} E_{i}\right)$ is the percent of the energy of $c_{i}(t)$ in the whole signal energy. Generally, the first $m$ IMFs containing the most of the faulty information are considered. These $m$ most informative selected IMFs, $c_{1}(t), c_{2}(t) \cdots c_{m}(t)$, include different frequency components and the energy distribution in the frequency domain of the rotating bearing vibration signal can be obtained as follows,

$\boldsymbol{T}=\left[\begin{array}{llll}H_{E N 1}(t) & H_{E N 2}(t) & \cdots & H_{E N m}(t)\end{array}\right]$

\subsection{Confidence value}

The proposed health assessment method is presented in Fig. 1. In order to provide an assessment of the bearing health, the confidence value $(\mathrm{CV})$, which ranges from zero to one, is calculated as health indicator to represent the bearing health state, with 1 indicating a perfect health condition and 0 indicating an unacceptable failure condition [28].

The $\mathrm{CV}$ is obtained from the EMD energy entropy through a SOM network, which provides a way of representing multi-dimensional features into a one or two-dimensional space [29]. Each neuron $i$ of the network is represented by an $n$-dimensional weight vector $m_{i}=$ $\left(m_{i 1}, m_{i 2}, \cdots, m_{i n}\right)^{T}$. The SOM is trained with data recorded during normal operation of healthy bearings. In the use of the trained SOM, for each input feature vector $\boldsymbol{T}$ there is a best matching unit (BMU), whose weight vector $m_{c}$ is the closest to the input vector and can be found in the trained SOM. The dis-tance between the input data and the weight vector of the BMU is defined as minimum quantization error (MQE), which actually quantizes how far the degradation condition is from the normal operation state [30]. The MQE can be calculated and converted into CV to represent the degradation state of the bearings as follows,

$$
\begin{aligned}
& \mathrm{MQE}=\left\|\boldsymbol{T}-\boldsymbol{m}_{B M U}\right\| \\
& \mathrm{CV}=\frac{c}{\sqrt{\mathrm{MQE}}+c}
\end{aligned}
$$




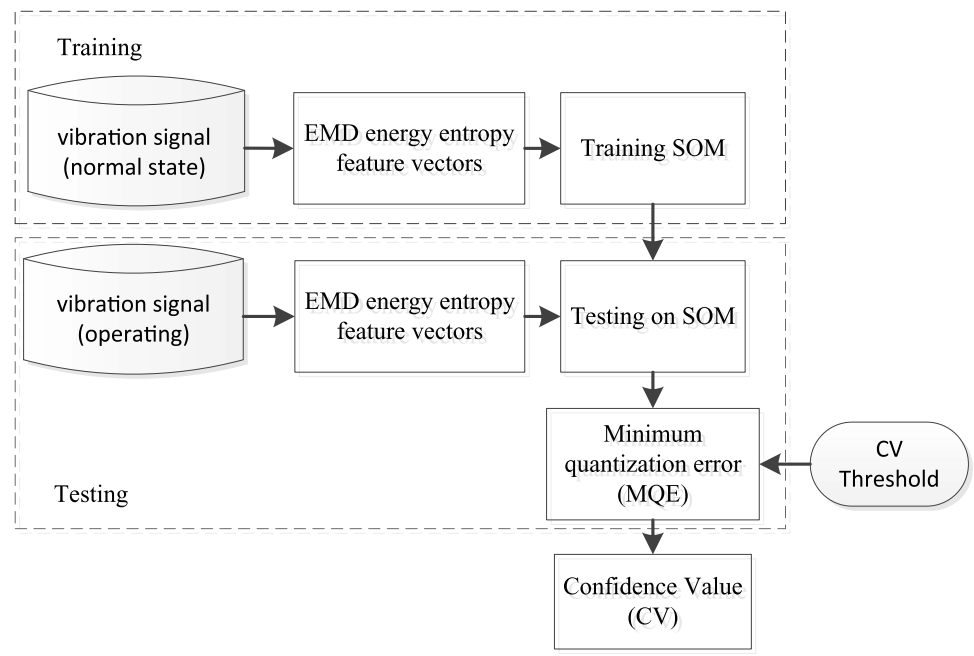

Fig. 1. Overview of the proposed health assessment method.

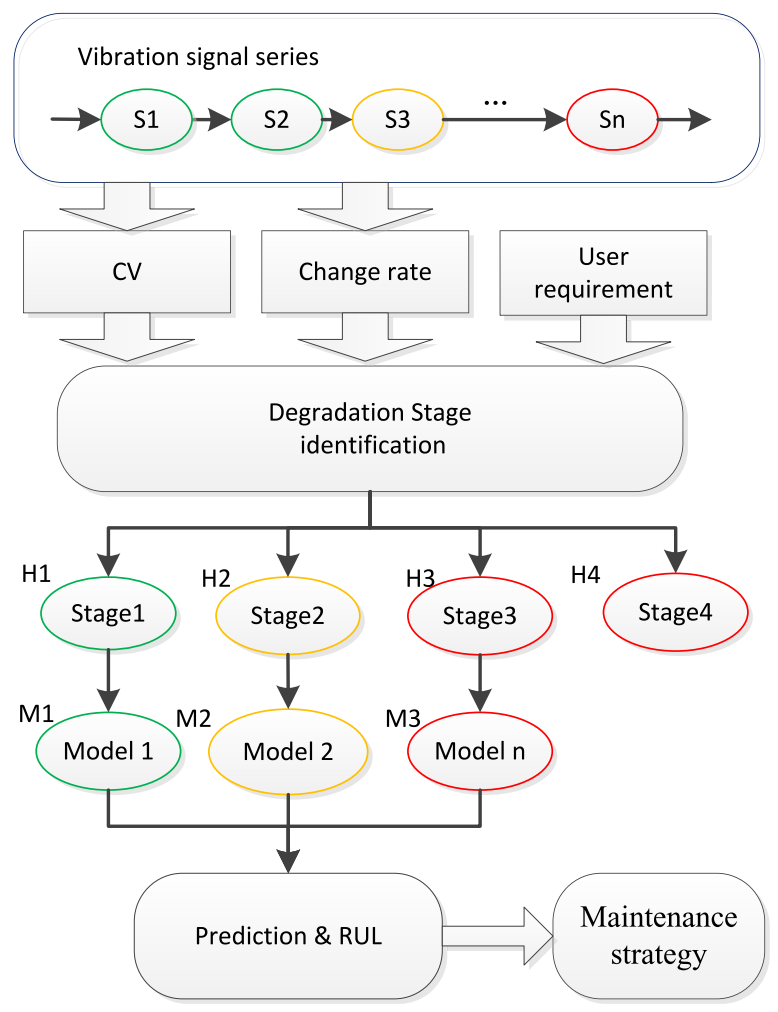

Fig. 2. Adaptive condition-based health trend prediction method scheme.

where $\boldsymbol{m}_{B M U}$ stands for the weight vector of the BMU of the input $\boldsymbol{T}$, and $c$ is a scale parameter determined by the MQE under normal conditions.

\section{Adaptive trend prediction method}

The procedure of the condition-based adaptive prognostics method is illustrated in Fig. 2. The bearing degradation process is described in four different stages, distinguished as normal, slight degradation, severe degradation and failure. Because the charac-teristics of the degradation process and the requirements of the prediction in those four stages differ, we use different prediction methods in each, as presented in Table 1. Firstly, the collected vi-bration signal data is used to calculate the CV by using the health assessment method described in Section 2. Then, the change rate of the CV value of the bearing is used to identify which degradation stage the bearing is currently operating in.

According to the characteristics of each stage, the prediction model should be adaptively selected. The selection rules are de-veloped based on the historical data. The goal of applying the adaptive prediction method is to achieve the desired prediction accuracy in each stage while keeping the computational complexity under control with respect to the practical requirements of the different stages.

\subsection{Normal stage}

In this stage, the bearing is in the normal state and the CV value is maintained at a high level. This is the stage in which the bearing operates in, during most of its life. Thus, there is no need to use a complex prediction algorithm in this stage. We simply collect the vibration signal at a relatively low sampling frequency and monitor the CV value: an incipient fault starts if the CV values exceed the degradation threshold whose setting depends on the user requirements, e.g. $\mathrm{CV}=0.8$, and the bearing enters the degradation region.

\subsection{Slight degradation stage}

In this stage, slight degradation has emerged and the $\mathrm{CV}$ values are continuously decreasing, but the bearing can still be used. In this situation, we should increase the frequency of monitoring the signal and activate the prediction model. Some data-driven mod-els, such as ANN and ARMA, can be applied to track the bearing conditions. In the application that follows, we have chosen, as an example, the Wavelet Neural Network (WNN) algorithm to predict the $\mathrm{CV}$ value. WNN is proposed as an alternative to feed forward neural networks, based on wavelet transform theory [30]. WNN has been shown to possess good properties of approximation and robustness [31]. In WNN, wavelet basis functions are used as node activation functions. In the application of this paper, it is designed as a three-layer structure with an input layer, a hidden layer, and an output layer. Fig. 3 shows the schematic diagram of the three-layer WNN, where $I_{n}^{k}$ is the $n$-th input CV value of the $k$-th data vector. Morlet wavelet, which is a widely used wavelet function, is taken as the activation function in the hidden layer.

Once the CV value is lower than the threshold or the change rate of the CV value increases significantly, the bearing is considered to be suffering from a severe degradation and entering the third stage, precisely the severe degradation stage. 
Table 1

Characteristics, requirements and prediction method for the four different stages.

\begin{tabular}{llllll}
\hline Stage & Type & CV criterion & Sampling frequency & Computing requirement & Prediction method \\
\hline S1 & Normal & $0.8<\mathrm{CV}<1$ & Low & Low computation time & Threshold monitoring \\
S2 & Slight degradation & $0.65<\mathrm{CV}<0.8$ & Medium & $\begin{array}{l}\text { Low computation time } \\
\text { Medium accuracy }\end{array}$ & WNN $^{\mathrm{a}}$ \\
& & & & High accuracy & GPR $^{\mathrm{b}}$ \\
S3 & Severe degradation & $0.6<\mathrm{CV}<0.65$ & High & High precision \\
& & & & Probability Output & \\
& & & RUL & - \\
S4 & Failure & $\mathrm{CV}<0.6$ & - & Repair & \\
\hline
\end{tabular}

\footnotetext{
a $\mathrm{WNN}=$ Wavelet Neural Network.
}

b GPR = Gaussian Process Regression.

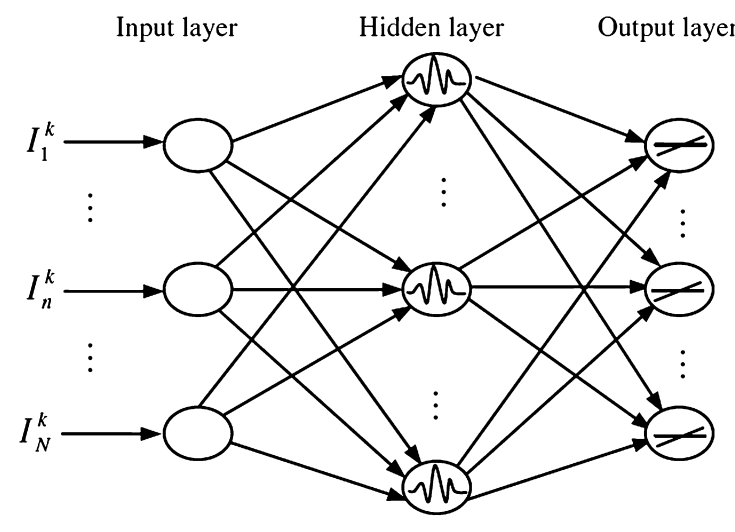

Fig. 3. Schematic diagram of the three-layer WNN.

\subsection{Severe degradation stage}

In this stage, the bearing is close to the end of its life. Although the bearing seems to work well, it may break down. In this situation, the accurate prediction of RUL is of utmost importance for the personnel to make maintenance decisions; then, one should further increase the sample frequency for monitoring and apply a more accurate prediction algorithm. This prediction algorithm should have a good capability to handle high data dimensionality and small sample size regression problems, because the bearing is degrading relatively fast. As an example, we chose the Gaussian Process Regression (GPR) algorithm for RUL prediction. GPR has high prediction accuracy and provides probabilistic outputs, which can reflect the confidence in the RUL prediction that is used to take the maintenance decisions [32]. As a probabilistic technique for nonlinear regression, it computes the posterior degradation estimates by constraining the prior distribution to fit the available training data $[33,34]$. For a new test input $\boldsymbol{x}^{*}$, we can establish a joint Gaussian prior distribution of the training output $\boldsymbol{y}$ and the test output $\boldsymbol{y}^{*}$ as follows,

$$
\left.\left.\begin{array}{lllcc}
\boldsymbol{y}^{*} & \sim N & 0, & C(\boldsymbol{X}, \boldsymbol{X})+\sigma_{n}^{2} \boldsymbol{I} & C\left(\boldsymbol{X}, \boldsymbol{x}^{*}\right) \\
\boldsymbol{y}^{*} & & C\left(\boldsymbol{X}, \boldsymbol{x}^{*}\right) & C\left(\boldsymbol{x}^{*}, \boldsymbol{x}^{*}\right)
\end{array}\right]\right)
$$

where $C(\boldsymbol{X}, \boldsymbol{X})$ is an $n$-order symmetric positive definite covariance matrix, $C\left(\boldsymbol{X}, \boldsymbol{x}^{*}\right)$ is the $n \times 1$ covariance matrix of the test input $\boldsymbol{x}^{*}$ and the training input $\boldsymbol{X}$, and $C\left(\boldsymbol{x}^{*}, \boldsymbol{x}^{*}\right)$ is the covariance matrix of the test input $\boldsymbol{x}^{*}$. Under the conditions of a given input $\boldsymbol{x}^{*}$ and the training set $\boldsymbol{D}=\left\{\left(\boldsymbol{x}_{i}, f\left(\boldsymbol{x}_{i}\right)\right) \mid i=1,2, \cdots n\right\}, \boldsymbol{x}_{i} \in \boldsymbol{X}$, the GP can calculate the test output $\boldsymbol{y}^{*}$ according to the posterior probability formula,

$\xi \boldsymbol{y}^{*} \mid \boldsymbol{x}^{*}, \boldsymbol{D} \sim N\left(\mu_{\boldsymbol{y}^{*}}, \sigma_{\boldsymbol{y}^{*}}^{2}\right)$

$\mu_{\boldsymbol{y}^{*}}=C \boldsymbol{x}^{*}, \boldsymbol{X} C(\boldsymbol{X}, \boldsymbol{X})+\sigma_{n}^{2} \boldsymbol{I}^{-1} \boldsymbol{y}=\sum_{i}^{n} \alpha_{i} C \boldsymbol{x}^{i}, \boldsymbol{x}^{*}$

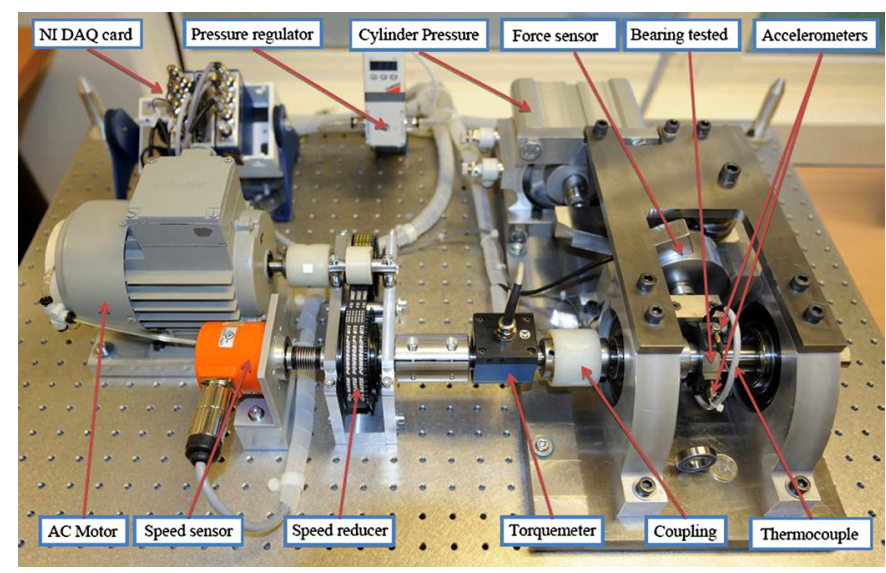

Fig. 4. Overview of the experimentation platform.

$\sigma_{\boldsymbol{y}^{*}}^{2}=C\left(\boldsymbol{x}^{*}, \boldsymbol{x}^{*}\right)-C^{T}\left(\boldsymbol{x}^{*}, \boldsymbol{X}\right)\left(C(\boldsymbol{X}, \boldsymbol{X})+\sigma_{n}^{2} \boldsymbol{I}^{-1} C \boldsymbol{x}^{*}, \boldsymbol{X}\right.$

where $\mu_{\boldsymbol{y}^{*}}, \sigma_{\boldsymbol{y}^{*}}$ are expectation and variance of $\boldsymbol{y}^{*}$ and $\alpha=$ $\left(C(\boldsymbol{X}, \boldsymbol{X})+\sigma_{n}^{2} \boldsymbol{I}\right)^{-1} y$ where $\boldsymbol{I}$ is the unit matrix. Therefore, the predicted value is a linear combination of the covariance function $C\left(\boldsymbol{x}^{i}, \boldsymbol{x}^{j}\right)$. Once the posterior distribution is obtained, it can be used to assess prediction values for the test data points. Typically, the squared exponential (SE) covariance function is used and the hyper-parameters in the covariance functions can be optimized from the training data by maximizing the marginal likelihood al-gorithm [35].

\subsection{Failure stage}

The failure stage is the last stage of a bearing life. The bearing may not be completely broken down but due to safety requirements or others, it should be replaced immediately when entering this stage. Also this final failure threshold is set according to the user requirements. In the example that follows, we set the threshold at $\mathrm{CV}=0.6$, with adequate safety margin to maximize the usage of the component while preventing complete failure.

\section{Experiment and analysis}

\subsection{Description of the experiment}

In order to validate the proposed adaptive prediction method, bearing run-to-failure test data were used, taken from the bearing Accelerated Life Tests (ALT) [36]. The overview of the experimentation platform is presented in Fig. 4. Two accelerometers are placed radially on the external race of the bearing in vertical and horizontal directions, respectively, and the load is applied to the bearing radially in horizontal direction. Vibration data are recorded from two channels for each bearing, at a sampling frequency of $25.6 \mathrm{kHz}$ every $10 \mathrm{~s}$. Seventeen bearings are tested in three different opera- 
Table 2

Bearing operation conditions.

\begin{tabular}{cllll}
\hline Dataset & $\begin{array}{l}\text { Load } \\
(\mathrm{N})\end{array}$ & $\begin{array}{l}\text { Speed } \\
(\mathrm{rev} / \mathrm{min})\end{array}$ & Training data & Testing data \\
\hline Dataset1 & 4000 & 1800 & $\begin{array}{l}\text { Bearing 1-1 Bearing 1-2 } \\
\text { Bearing 1-3 Bearing 1-4 } \\
\text { Bearing 1-5 }\end{array}$ & $\begin{array}{l}\text { Bearing 1-6 } \\
\text { Bearing 1-7 }\end{array}$ \\
Dataset2 & 4200 & 1650 & $\begin{array}{l}\text { Bearing 2-1 Bearing 2-2 } \\
\text { Bearing 2-3 Bearing 2-4 }\end{array}$ & Bearing 2-6 \\
& & & $\begin{array}{l}\text { Bearing 2-5 } \\
\text { Bearing 2-7 }\end{array}$ & \\
\hline
\end{tabular}

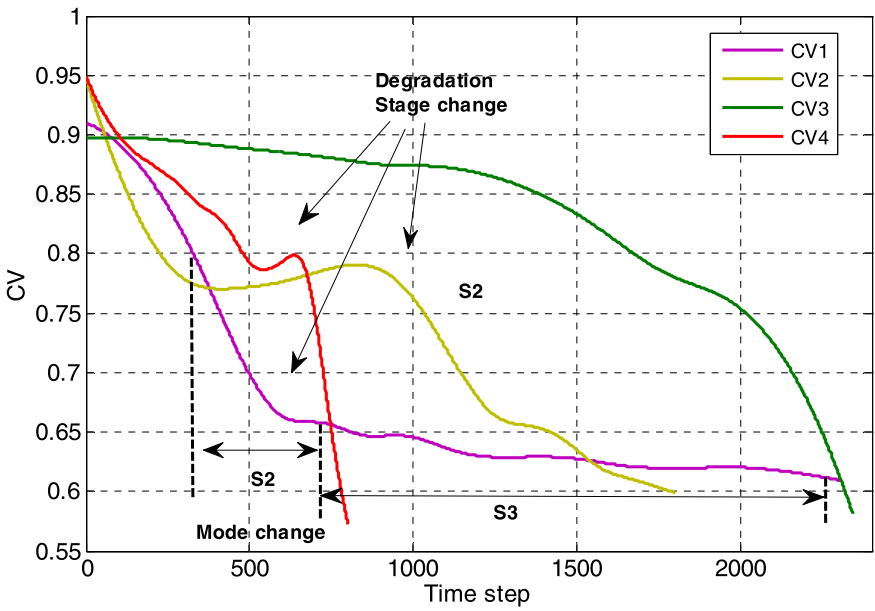

Fig. 5. Four CV curves of the training data after health assessment.

tion conditions, as shown in Table 2. Twelve bearing data are taken for training, and the remaining five bearing sets of data are used for testing.

\subsection{Adaptive prediction}

By applying the EMD method, the vibration signals are decomposed into several IMFs. The first five IMFs $C_{1}(t), C_{2}(t), \ldots, C_{5}(t)$ include the most relevant degradation information, and are arranged from high to low frequency. The corresponding energy entropies are calculated and the feature vectors $\boldsymbol{T}$ are obtained according to (2)-(4). The first 50 feature vectors of the training data when the bearings are in the normal state are used to train the SOM. After training, the whole life data of the whole 17 bearings are used for testing and the CV curves are obtained by using (5)-(6).

Due to the limited space, only four typical CV curves of the training data are given in Fig. 5. Some obvious stage changes can be found in CV1, CV2 and CV4. Once the CV curves have been obtained, the corresponding degradation rates can be calculated. A higher degradation rate represents a rapid deterioration, whereas a lower rate indicates a slow evolving health state. The varying degradation modes suggest that the adaptive prediction model should be applied.

An example of adaptive prediction for Bearing 3-3 is illustrated in Fig. 6. The CV curve is obtained and three degradation stages can be identified as shown in Fig. 6(a). In the first stage of the $\mathrm{CV}$ curve, a slight degradation can be found when the $\mathrm{CV}$ value reaches the threshold 0.8 , but there is no need to predict the RUL due to the relatively high $\mathrm{CV}$ value. $\mathrm{CV}$ decreases as bearing performance deteriorates continuously, then turns into a stable period up to time $300 \mathrm{~s}$. A slight degradation stage can be identified. Thus, the WNN model is used to predict the degradation process in this stage. Seven past $C V$ values are used to predict the next, one time step ahead, and 15 data vectors are used for training.

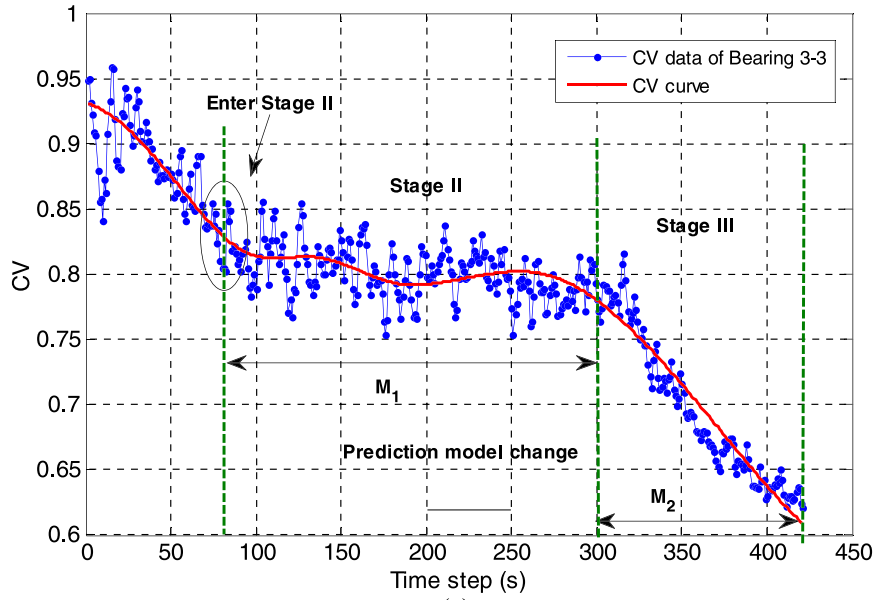

(a)

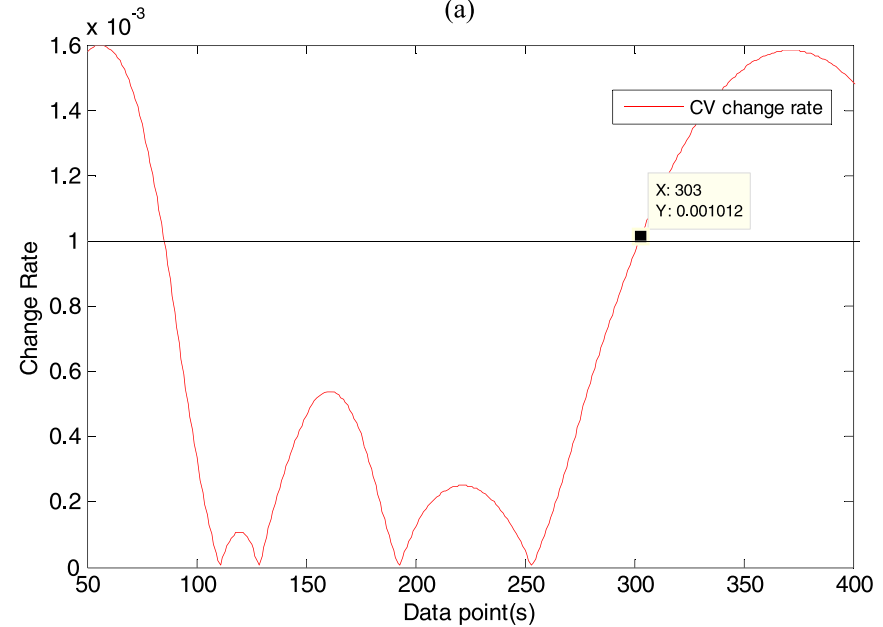

(b)

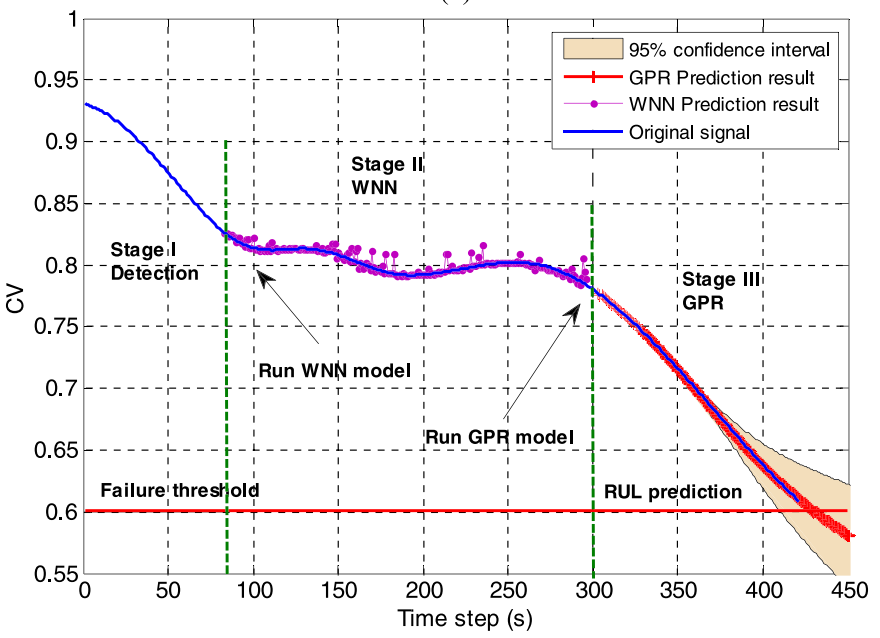

(c)

Fig. 6. Adaptive prediction of Bearing 3-3.

Fig. 6(b) shows the CV change rate of the Bearing 3-3. After time step $303 \mathrm{~s}$, the degradation mode of the bearing gradually changes into rapid degradation. The GPR model is selected to estimate the RUL. The prediction result at time $330 \mathrm{~s}$ is presented in Fig. 6(c), 20 data points are recorded and used to train the GPR model, the prediction result is $431 \mathrm{~s}$, whereas the actual final time is $427 \mathrm{~s}$. The relative prediction error of RUL is $4.1 \%$, calculated by using the following formula,

$E r=\frac{{\operatorname{ActR} U L_{i}-\operatorname{PredR} U L_{i}}_{A c t R U L_{i}}}{A 100 \%}$ 
Table 3

Testing results of the three prediction methods.

\begin{tabular}{|c|c|c|c|c|c|c|}
\hline \multirow[t]{2}{*}{ Bearing } & \multicolumn{2}{|c|}{ Adaptive prediction model } & \multicolumn{2}{|c|}{ WNN model } & \multicolumn{2}{|c|}{ GPR model } \\
\hline & Error \% & Time (s) & Error \% & Time $(s)$ & Error \% & Time (s) \\
\hline Bearing 1-6 & 0.1960 & 0.3434 & 0.3009 & 0.1596 & 0.0579 & 0.5858 \\
\hline Bearing 1-7 & 0.1413 & 0.4573 & 0.3073 & 0.2019 & 0.0655 & 0.5742 \\
\hline Bearing 2-6 & 0.2592 & 0.4111 & 0.2691 & 0.4113 & 0.0579 & 0.4091 \\
\hline Bearing 2-7 & 0.8460 & 0.3494 & 1.0682 & 0.3663 & 0.2353 & 0.3031 \\
\hline Bearing 3-3 & 0.2968 & 0.2581 & 0.3993 & 0.2608 & 0.0929 & 0.2528 \\
\hline
\end{tabular}

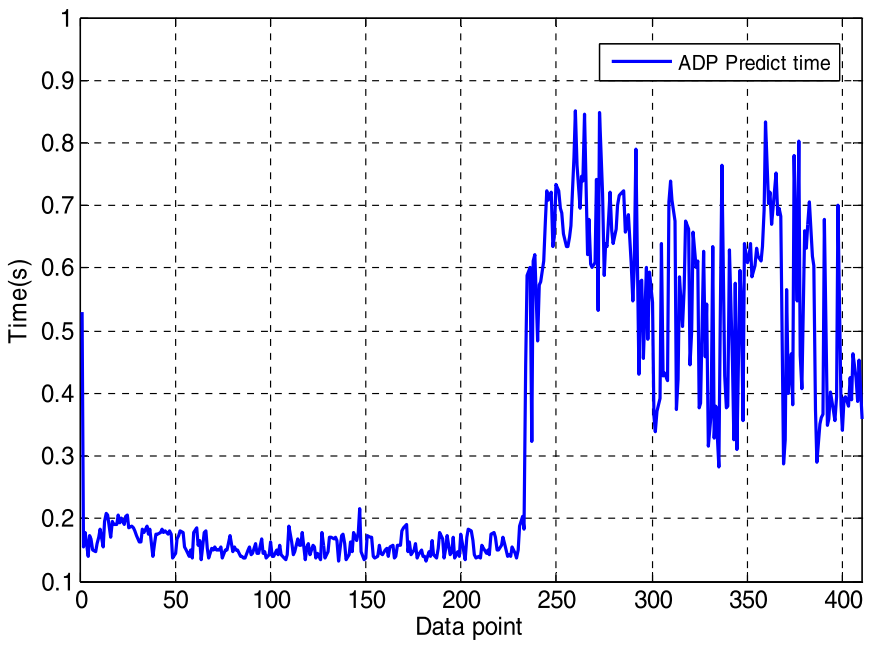

(a)

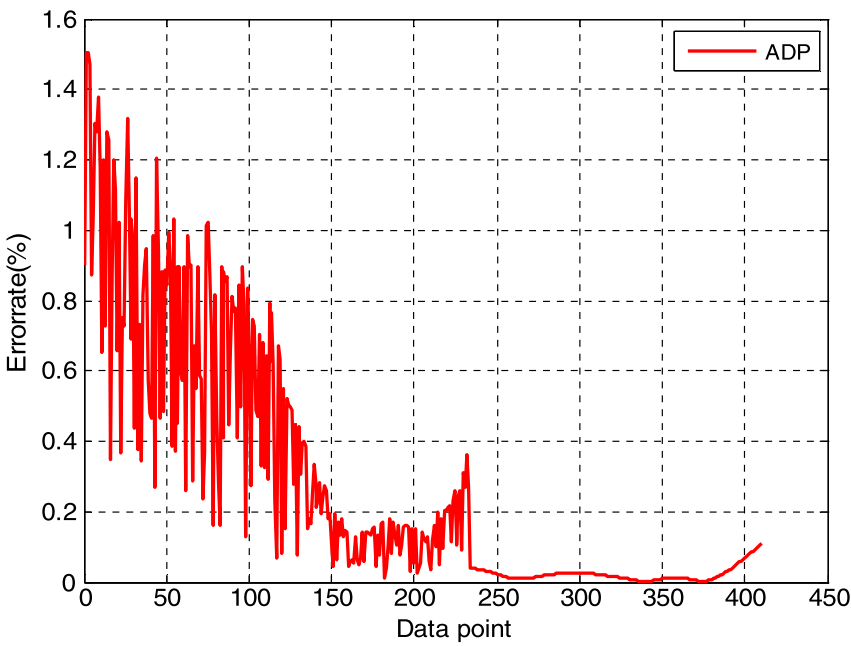

(b)

Fig. 7. Computation time and relative error of the adaptive prediction method

where $A c t R U L_{i}$ is the actual RUL and PredRUL $L_{i}$ is the estimation of RUL.

\subsection{Performance analysis and discussion}

According to previous research [37], the limitation of GPR is in the expensive computations needed for inverting the covariance matrix $\left(\boldsymbol{K}+\sigma_{n}^{2} \boldsymbol{I}\right)^{-1}$, which yields a cost of $\mathrm{O}\left(N^{3}\right)$, and for the mean prediction, which has a cost of $\mathrm{O}(N)$, where $N$ is the number of data points. From our experience in the experiment, to get high accuracy GPR indeed needs a substantially high computational cost during both learning and prediction. Although the GPR algorithm can get high prediction accuracy, it can result in significant computing resources also in the early or mid-degradation stages. In contrast to GPR, the WNN has approximately a complexity of $\mathrm{O}(n m N)$ for training the network parameters, where $m$ is the number of input nodes, $n$ is the output nodes, and $N$ is the number of data [38]. In general, WNN consumes little computational resources during the prediction process.

Given the above, in the proposed adaptive method, we use the less computationally complex WNN method in the early degra-dation stages and apply the high prediction accuracy, relatively high computational cost GPR method in the late degradation stage. This ensures overall satisfactory prediction accuracy and reasonable consumption of computing resources suitable to the requirements.

To further validate the performance of the proposed method, WNN and GPR are applied also separately throughout all degradation stages. The testing results of the three prediction methods are presented in Table 3. Five bearings are used for this analysis. It is easy to see that the WNN method employs less computing time, but does not achieve as much accuracy as the GPR method.

An example of the computation time and relative error of the adaptive prediction method is illustrated in Fig. 7. The prediction algorithm is changed after time $303 \mathrm{~s}$ (Data point 233 in Fig. 7): as discussed in Section 3, low computation time and medium accuracy are important in stage 2; the computation time for the prediction is relatively low by using WNN, as shown in Fig. 7(a); however, when the bearing enters into stage 3 , there is a requirement of RUL prediction accuracy: then, the GPR method is applied, with more computational cost in exchange for high accuracy, as shown in Fig. 7(b).

\section{Conclusion}

In this paper, we have proposed a condition-based adaptive method for bearing health trend analysis and RUL prediction. The EMD energy entropy and SOM are applied to calculate the CV for representing the health state of the bearings. Then, the CV value and the CV change rate are used to identify the current stage of the degradation dynamic process, and the correspondingly adequate prognostic models are selected to estimate the health trend and RUL. Features of the four stages, including characteristics, criteria requirements and prediction methods, are discussed. A case study concerning data from 17 test bearings has been analyzed to verify the feasibility of the method, with WNN and GPR as tailored models for the different degradation stages.

From the experiment results and analysis, the proposed method offers a best trade-off between the efficiency and consume. Compared with the traditional single method like WNN, the proposed method adaptively adjusts the prediction algorithm according to the degraded condition and enhances the accuracy when entering severely degraded stage. It can be concluded that the proposed method can provide high prediction accuracy while effectively controlling the computational complexity and CPU resource consumption. It guides also a strategy for setting the sampling frequency and selecting the appropriate prediction method, which is useful for application in real industrial systems. 
Furthermore, alternative data-driven models, such as autoregressive moving average (ARMA) and support vector regression (SVR), are good candidate algorithms for the prediction in the different degradation stages: by selecting the best model in each stage, the proposed adaptive method can provide the required accuracy within the time and computational requirements of the actual application.

\section{Acknowledgments}

The authors are highly thankful for the financial support of National Key Basic Research Program of China under grant No. 2014CB744904, National Natural Science Foundation of China under grant No. 61304111 and No. 71231001, Fundamental Research Funds for the Central Universities under grant Nos. YWF-14-KKX001 and YWF-13-JQCJ, China.

\section{References}

[1] A.K.S. Jardine, D. Lin, D. Banjevic, A review on machinery diagnostics and prognostics implementing condition-based maintenance, Mech. Syst. Signal Process. 20 (7) (2006) 1483-1510.

[2] J. Cheng, Y. Yang, Y. Yang, A rotating machinery fault diagnosis method based on local mean decomposition, Digit. Signal Process. 22 (2012) 356-366.

[3] A.R. Messina, V. Vittal, G.T. Heydt, T.J. Browne, Nonstationary approaches to trend identification and denoising of measured power system oscillations, IEEE Trans. Power Syst. 24 (2009) 1798-1807.

[4] Y. Pan, J. Chen, X. Li, Bearing performance degradation assessment based on lifting wavelet packet decomposition and fuzzy c-means, Mech. Syst. Signal Process. 24 (2010) 559-566.

[5] C. Hu, B.D. Youn, P. Wang, J. Taek Yoon, Ensemble of data-driven prognostic algorithms for robust prediction of remaining useful life, Reliab. Eng. Syst. Saf. 103 (2012) 120-135.

[6] G.F. Bin, J.J. Gao, X.J. Li, B.S. Dhillon, Early fault diagnosis of rotating machinery based on wavelet packets-empirical mode decomposition feature extraction and neural network, Mech. Syst. Signal Process. 27 (2012) 696-711.

[7] F. Di Maio, K.L. Tsui, E. Zio, Combining Relevance Vector Machines and exponential regression for bearing residual life estimation, Mech. Syst. Signal Process. 31 (2012) 405-427.

[8] Y. Peng, M. Dong, M.J. Zuo, Current status of machine prognostics in conditionbased maintenance: a review, Int. J. Adv. Manuf. Technol. 50 (1-4) (2010) 297-313.

[9] A. Heng, S. Zhang, A.C. Tan, J. Mathew, Rotating machinery prognostics: state of the art, challenges and opportunities, Mech. Syst. Signal Process. 23 (2009) 724-739.

[10] X. Si, W. Wang, C. Hu, D. Zhou, Remaining useful life estimation-a review on the statistical data driven approaches, Eur. J. Oper. Res. 213 (2011) 1-14.

[11] T. Benkedjouh, K. Medjaher, N. Zerhouni, S. Rechak, Remaining useful life estimation based on nonlinear feature reduction and support vector regression, Eng. Appl. Artif. Intell. 26 (2013) 1751-1760.

[12] Z. Wei, T. Tao, D. ZhuoShu, E. Zio, A dynamic particle filter-support vector regression method for reliability prediction, Reliab. Eng. Syst. Saf. 119 (2013) 109-116.

[13] A. Widodo, B.S. Yang, Machine health prognostics using survival probability and support vector machine, Expert Syst. Appl. 38 (7) (2011) 8430-8437.

[14] D. Liu, Y. Peng, J. Li, X. Peng, Multiple optimized online support vector regression for adaptive time series prediction, Measurement 46 (8) (2013) 2391-2404.

[15] H. Liao, Z. Tian, A framework for predicting the remaining useful life of a single unit under time-varying operating conditions, IIE Trans. 45 (9) (2013) 964-980.

[16] L. Liao, F. Kottig, Review of hybrid prognostics approaches for remaining useful life prediction of engineered systems, and an application to battery life prediction, IEEE Trans. Reliab. 63 (1) (2014) 191-207.

[17] J. Sun, H. Zuo, W. Wang, et al., Prognostics uncertainty reduction by fusing online monitoring data based on a state-space-based degradation model, Mech. Syst. Signal Process. 45 (2) (2014) 396-407.

[18] A. Soualhi, H. Razik, G. Clerc, et al., Prognosis of bearing failures using hidden Markov models and the adaptive neuro-fuzzy inference system, IEEE Trans. Ind. Electron. 61 (6) (2014) 2864-2874.

[19] B.L. Song, J. Lee, Framework of designing an adaptive and multi-regime prognostics and health management for wind turbine reliability and efficiency im-provement, Int. J. Adv. Comput. Sci. Appl. 4 (2) (2013) 142-149.

[20] J. Lee, F. Wu, W. Zhao, et al., Prognostics and health management design for rotary machinery systems-reviews, methodology and applications, Mech. Syst. Signal Process. 42 (1) (2014) 314-334.
[21] P. Baraldi, F. Mangili, E. Zio, Investigation of uncertainty treatment capability of model-based and data-driven prognostic methods using simulated data, Reliab. Eng. Syst. Saf. 112 (2012) 94-108.

[22] F. Wu, T. Wang, J. Lee, An online adaptive condition-based maintenance method for mechanical systems, Mech. Syst. Signal Process. 24 (8) (2010) 2985-2995.

[23] E. Zio, Prognostics and health management of industrial equipment, in: Seifedine Kadry (Ed.), Diagnostics and Prognostics of Engineering Systems: Methods and Techniques, 2012, pp. 333-356.

[24] L. Liao, J. Lee, Design of a reconfigurable prognostics platform for machine tools, Expert Syst. Appl. 37 (1) (2010) 240-252.

[25] S. Hong, Z. Zhou, Remaining useful life prognosis of bearing based on Gauss process regression, in: International Conference on Biomedical Engineering and Informatics, BMEI, IEEE, 2012, pp. 1575-1579.

[26] T.L Xu, X.Z. Lang, X.Y. Zhang, X.C. Pei, The research based on empirical mode decomposition in bearing fault diagnosis, Appl. Mech. Mater. 103 (2012) 225-228.

[27] Y. Lei, J. Lin, Z. He, M.J. Zuo, A review on empirical mode decomposition in fault diagnosis of rotating machinery, Mech. Syst. Signal Process. 35 (2013) 108-126.

[28] H. Qiu, J. Lee, J. Lin, G. Yu, Robust performance degradation assessment methods for enhanced rolling element bearing prognostics, Adv. Eng. Inform. 17 (2003) 127-140.

[29] J. Hu, L. Zhang, W. Liang, Dynamic degradation observer for bearing fault by MTS-SOM system, Mech. Syst. Signal Process. 36 (2) (2012) 385-400.

[30] J. Yu, A hybrid feature selection scheme and self-organizing map model for machine health assessment, Appl. Soft Comput. 11 (2011) 4041-4054.

[31] P. Wang, G. Vachtsevanos, Fault prognostics using dynamic wavelet neural networks, Artif. Intell. Eng. Des. Anal. Manuf. 15 (4) (2001) 349-365.

[32] Y. Lei, Z. He, Y. Zi, EEMD method and WNN for fault diagnosis of locomotive roller bearings, Expert Syst. Appl. 38 (6) (2011) 7334-7341.

[33] S. Saha, B. Saha, A. Saxena, K. Goebel, Distributed prognostic health management with Gaussian process regression, in: IEEE Aerospace Conference, 2010, pp. 1-8.

[34] K. Liu, B. Liu, C. Xu, Intelligent analysis model of slope nonlinear displacement time series based on genetic-Gaussian process regression algorithm of combined kernel function, Chin. J. Rock Mech. Eng. 10 (2009) 2128-2134.

[35] C.E. Rasmussen, C.K.I. Williams, Gaussian Processes for Machine Learning, MIT Press, Cambridge, MA, 2006.

[36] P. Nectoux, R. Gouriveau, K. Medjaher, E. Ramasso, B. Chebel-Morello, N. Zerhouni, C. Varnier, PRONOSTIA: an experimental platform for bearings accelerated degradation tests, in: IEEE International Conference on Prognostics and Health Management, 2012, pp. 1-8.

[37] D. Nguyen-Tuong, J. Peters, Local Gaussian process regression for real-time model-based robot control, in: International Conference on Intelligent Robots and Systems, 2008, pp. 380-385.

[38] Y. Shao, K. Nezu, Prognosis of remaining bearing life using neural networks, Proc. Inst. Mech. Eng., Part I, J. Syst. Control Eng. 214 (3) (2000) 217-230.

Sheng Hong was born in China, in 1981. He received his master degree and doctoral degree in communication and information system from Beihang University in 2005 and 2009, respectively. He is now a graduate student advisor in the School of Reliability and System Engineering of Beihang University. His recent interests include signal processing, information system modeling, prognostics and heath management.

Zheng Zhou was born in China, in 1989. He received his master degree in Reliability and System Engineering from Beihang University in 2014. He is now an engineer of Systems Engineering Research Institute, CSSC.

Enrico Zio is Director of the Chair in Complex Systems and the Energetic Challenge of Ecole Centrale Paris and Supelec, Director of the Graduate School of the Politecnico di Milano, full professor of Computational Methods for Safety and Risk Analysis, adjunct professor in Risk Analysis at the University of Stavanger, Norway and at University Santa Maria, Chile, and invited lecturer and committee member at various Master and $\mathrm{PhD}$ Programs in Italy and abroad.

Wenbin Wang was born in China. He received his $\mathrm{PhD}$ in Operational Research and Applied Statistics from University of Salford in 1992. He is the Dean of Dongling School of Economics and Management, University of Science and Technology Beijing, Beijing, China. His recent interests include maintenance and reliability modeling, degradation processes and stochastic modeling, residual life estimation of complex system. 